\title{
Analysis and Compensation of PEM Fuel Cell Instabilities in Low-Frequency EIS Measurements
}

\author{
Giovanni Dotelli, Roberto Ferrero, Paola Gallo Stampino, and Saverio Latorrata
}

\begin{abstract}
This paper analyzes possible humidity-related instabilities of polymer electrolyte membrane (PEM) fuel cell (FC) operation that affect electrochemical impedance spectroscopy measurements. The compensation of ohmic resistance variations due to such instabilities, achieved using the well known multisine excitation technique with properly chosen high-frequency harmonic components, is proposed here as a new approach to improve low-frequency measurements. Additionally, this method allows a more accurate determination of equivalent circuit parameters identified from impedance spectra and their uncertainties taking into account correlation between impedances at different frequencies, and thus avoiding uncertainty overestimation of low-frequency parameters (such as transport and activation equivalent resistances) that is likely to occur with classic measurement techniques. The proposed method is experimentally validated on a single PEM FC.
\end{abstract}

Index Terms-Frequency response, fuel cell (FC), impedance spectroscopy, measurement uncertainty.

\section{INTRODUCTION}

$\mathbf{E}$ LECTROCHEMICAL impedance spectroscopy (EIS) is one of the most common techniques employed to study the behavior of electrochemical devices, such as fuel cells (FCs) [1]-[3], and in particular, it is widely employed for polymer electrolyte membrane (PEM) FC characterization. Its ability to distinguish the different electrochemical processes that define the FC behavior on different time scales makes it a very powerful tool for both research in the design phase and diagnostics during operation. Several works can be found in the literature, in which this technique is employed to identify FC models (see [4]-[7]), to investigate the FC response in particular conditions, useful in the design phase (see [8]-[13]), and to identify nonproper operation and fault conditions (see [14]-[17]).

Depending on the aim of the analysis, measured impedance spectra can be fitted by suitable models to identify relevant parameters related to the features under investigation. As an

Manuscript received October 7, 2013; revised December 24, 2013; accepted December 25, 2013. Date of publication January 15, 2014; date of current version June 5, 2014. The Associate Editor coordinating the review process was Dr. Subhas Mukhopadhyay.

G. Dotelli, P. G. Stampino, and S. Latorrata are with the Department of Chemistry, Materials, and Chemical Engineering "G. Natta," Politecnico di Milano, Milan 20133, Italy (e-mail: giovanni.dotelli@polimi.it; paola.gallo@polimi.it; saverio.latorrata@polimi.it).

R. Ferrero is with the Department of Energy, Systems, Land and Constructions, University of Pisa, Pisa 56122, Italy (e-mail: roberto.ferrero@for.unipi.it).

Color versions of one or more of the figures in this paper are available online at http://ieeexplore.ieee.org. example, the high-frequency limit impedance is usually interpreted as the equivalent ohmic resistance of the $\mathrm{FC}$, whose value is a good indicator of the membrane humidity and it can be used for diagnostic purposes [16]-[18].

An accurate measurement uncertainty evaluation for such parameters is always important to correctly recognize whether an observed variation of their values is significant or not, thus avoiding possible misinterpretation of measurement results. While the type-B uncertainty associated with instrumentation is usually reasonably low even with low-cost instrumentation, a type-A evaluation is likely to provide a higher uncertainty value, especially at low frequency, due to uncontrollable variability of FC internal conditions, mainly related to membrane humidity [19].

Such great impedance variability appearing at low frequency can be explained considering that the low-frequency response is determined by several processes, thus it depends on several quantities, each of them characterized by its own variability. For this reason, when equivalent circuit parameters are identified from impedance spectrum fitting, the uncertainty of the parameters associated with the low-frequency part of the circuit could be greatly overestimated, unless correlation between impedance measurements at different frequencies is correctly considered.

However, such correlation analysis is often quite critical, since uncontrolled parameter variations occur also during the acquisition of a single impedance spectrum, particularly when the frequency range is extended down to very low frequencies, requiring longer acquisition time. This means that the correlation between impedance measurements at different frequencies cannot be correctly evaluated if they are measured at different times, as in classic frequency response analysis employing single-sine excitation signals with stepwise frequency sweep. Moreover, at low frequency, the time scale of the abovementioned variations might become comparable with the period of the excitation signal, meaning that the system cannot be considered in stationary conditions either during the single impedance measurement at that frequency.

Nonstationary systems introduce distortions in the output waveform that can lead to completely meaningless impedance measurements when the time scale of system variations is comparable with the excitation signal period. For this reason, some papers in the literature state that accurate impedance measurements at low frequency are not possible because of instabilities in FC operation (see [8], [15]). However, depending on the cause of the system nonstationarity, a correct interpretation of EIS measurements might still be 
possible using more complex measurement techniques and mathematical tools for data processing. Possible solutions have already been proposed (see [20], [21]), applicable in particular conditions. A deeper review of the state of the art on this topic is presented in Section II.

Within this framework, the aim of this paper is to present a possible method to compensate humidity-related instabilities of PEM FC operation appearing as uncontrollable slow variations of the equivalent ohmic resistance (on time scales from seconds to tens of seconds). This compensation is carried out in the time domain by means of a continuous ohmic resistance measurement obtained by a high-frequency excitation signal superimposed to the main perturbation signal employed for EIS. In addition to improving low-frequency impedance measurements by eliminating the abovementioned waveform distortions, this method also allows a more correct uncertainty evaluation of low-frequency equivalent circuit parameters identified from impedance measurements.

After a brief analysis of typical humidity-related instabilities occurring on a PEM FC and the different ways in which they affect impedance measurements (Section III), the proposed method is presented in Section IV, and the results of the experimental validation on a single PEM FC are reported in Section V.

\section{EIS MEASUREMENTS ON NONSTATIONARY SYSTEMS}

The validity of a frequency response model of a system relies on three main assumptions that the system must satisfy: linearity, causality, and stationarity. Strictly speaking, none of these are generally verified by an FC, which is an active nonlinear electrochemical device. Nevertheless, from a practical point of view, linearity can be ensured by applying small perturbations to the system, and causality and stationarity can often be considered satisfied, provided that the system has reached a stable equilibrium condition.

Unfortunately, however, in several cases, the equilibrium reached by the FC after all transients associated with relaxation phenomena have ended is not stable and significant variations of the cell internal conditions still occur (a typical example concerns the membrane humidity, as discussed in the next section). In this case, the validity of the stationarity assumption depends on the time scale of these variations compared with the measurement time, which typically ranges from tens of seconds to several minutes, depending on the chosen frequency range and desired accuracy. If the system does not vary much during this time (so that no significant distortion appears in the response waveform), it can be considered in quasi-stationary conditions and the Fourier analysis can be applied without significant errors.

Classic EIS analysis employs single-sine excitation signals, superimposed to the FC current (or voltage) usually by properly controlled electronic loads. The frequency is changed after each impedance measurement to acquire the whole spectrum in the desired frequency range and with the desired number of points. This approach has two important advantages. First, it allows the highest signal-to-noise ratio for each frequency, as the whole signal power is concentrated in one harmonic component, and therefore it allows to find the best tradeoff between the requirement of linear response (i.e., small perturbation) and low uncertainty of the impedance measurement. Second, it is easily implemented without the need of memory- and time-consuming algorithms for data processing, e.g., the lock-in amplification technique is able to provide accurate measurements with high noise immunity using simple and low-cost instrumentation [22].

On the other hand, the main drawback of the singlesine technique is the long measurement time required to measure the whole impedance spectrum, which becomes a critical problem for a nonstationary system, according to what stated above. To reduce the measurement time, techniques based on multisine signals were developed [23]-[27], so that impedances at different frequencies are simultaneously measured. Alternative solutions to the multisine signals involve other periodic waveforms, such as chirp signal, maximum length binary sequence, and discrete binary sequence [28]. It is worth mentioning that the use of periodic waveforms is generally preferred as it avoids leakage errors, but also aperiodic waveforms can be used, such as white noise [29]. Finally, another approach to reduce the measurement time is the identification of impedance values by time-domain fitting of the current and voltage waveforms, so that an integer number of periods is not required [30].

The minimization of the measurement time is useful if it allows to consider the system in quasi-stationary conditions. However, when this approximation is not justified because of significant system variations during a single-frequency impedance measurement, the Fourier transform cannot be applied and more complex mathematical tools are required. Several approaches are known for EIS measurement validation, allowing to recognize the experimental data affected by nonstationary conditions. Among these, the most commonly employed are the Kramers-Kronig transforms [31], [32] and the comparison between input and output signal spectra to detect the appearance of nonexcited harmonics in the output spectrum [26], [33].

Once nonstationary conditions have been recognized, correction methods need to be applied, when possible. When an impedance spectrum does not meet the stationarity condition because the impedances at different frequencies are measured at different times (with classic single-sine signals), but each impedance measurement can be considered valid, a simple correction method is based on time-domain interpolation of several spectra acquired consecutively, so that the impedance spectrum at a single instant can be estimated [34], [35].

On the other hand, when nonstationarity affects the validity of the single impedance measurement obtained by classic Fourier analysis, other approaches are necessary. In [34], a new mathematical operator is defined, called rotating Fourier transform, able to estimate instantaneous impedance values. On the contrary, a different approach still based on classic Fourier transform is proposed in [21]. In this case, the time dependence of system parameters is included in the model using proper functions as time-varying gains, and the experimental output spectrum is fitted by this model in the frequency domain, so that the instantaneous impedance can be identified. Finally, 
in [20], a two-cell method is proposed for a time-domain compensation of nonstationarity due to relaxation phenomena, based on the measurement of such relaxation transients on another identical system, not subjected to the ac perturbation.

Similarly to the last mentioned approach, the basis of the method proposed in this paper for PEM FCs is a time-domain compensation of the cause of the nonstationary response. However, in this case, it is not related to relaxation transients, but to ohmic resistance variations due to humidity-related instabilities, and its compensation does not require another identical FC. In more details, the idea is to remove the voltage drop associated with the time-varying ohmic resistance from the output voltage, and therefore to identify the impedance of the remaining part of the equivalent circuit (to which the stationarity hypothesis applies), using classic Fourier analysis.

Before analyzing the details of the measurement method, a brief explanation of FC humidity-related instabilities and their effect on impedance measurements is presented in the next section.

\section{AnAlysis of Low-FreQUenCy INSTABILITIES}

The membrane humidity is a very important parameter for PEM FC operation, particularly in the current technologies, which mainly utilize perfluorosulfonic acid (PFSA) membranes (Nafion is the prototypical example) [36]. Indeed, PFSA membrane conductivity is very sensitive to humidity content and this, in turn, has an influence on the efficiency of the electrochemical reactions [37] because it strongly affects the equivalent ohmic resistance, which is mainly governed just by the membrane ionic conductivity, being the resistance of electronic conductors (such as gas diffusion layer and catalyst layer) and contact resistances typically negligible [9].

Membrane humidity is generally guaranteed by humidification of inlet gases and by the water generated at the cathode by the oxygen reduction reaction; however, during cell operation, there is a massive water movement from anode to cathode (by electro-osmotic drag) and from cathode to anode (by water concentration gradient driven back diffusion), without considering pressure driven hydraulic permeation in the case of pressure gradient between the anode and cathode. In view of such a complex interplay of water transport mechanisms, it is not easy to control the membrane water content from the external control variables, such as input gas humidity and temperature. For this reason, apparently random variations of the membrane humidity (and, consequently, of the ohmic resistance) are likely to occur during FC operation, particularly in low-humidity conditions.

The conduction mechanism in PFSA membranes may be of help to understand these variations. In general, proton transport in PFSA membranes occurs in water channels through complex forms: protons are associated to water molecules to form species like hydronium ions $\left(\mathrm{H}_{3} \mathrm{O}^{+}\right)$. Accordingly, two conductivity mechanisms are possible: the direct movement of water-proton complex species (vehicle mechanism) through the channels or the so-called Grotthuss or hopping mechanism, where proton-accepting chemical species, such as water or phosphoric acid [38], form a bridge for protons that jump

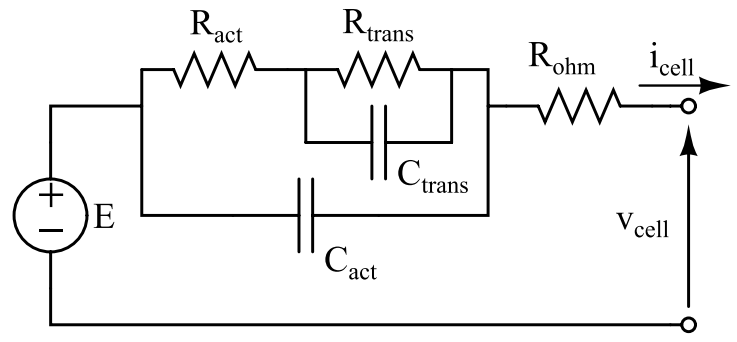

Fig. 1. Simplified linear equivalent circuit of a typical PEM FC.

from one species to the nearest one. The two mechanisms may be concomitant, but the first one is more accredited to be the one occurring in low degrees of hydration, whereas the second one at high levels of hydration. Anyway, in both cases, water is necessary for proton transport and water channels within the polymer should create a percolation path between the electrodes.

In fully humidified conditions, any path can conduct the protons, so the membrane conductivity is optimal, but when water saturation decreases more and more paths become useless. Then, in such condition, water diffusion through them may be the controlling factor of changes in ohmic resistance, even in apparently pseudostationary operating conditions, i.e., without any variation of external parameters, such as temperature or inlet gas humidity. A rough estimate of the time scale of water front advancement in the membrane can be done: the water concentration gradient within the membrane depends on the water diffusion coefficient $(D)$ and the membrane thickness $(L)$. Then, considering typical $D\left(10^{-11}-10^{-10} \mathrm{~m}^{2} / \mathrm{s}\right)$ and $L$ values $\left(10-10^{2} \mu \mathrm{m}\right)$, the time scale of the process $\left(L^{2} / D\right)$ is in the order of $10-10^{2} \mathrm{~s}$ [39], so it is comparable with the measurement time of typical impedance spectra acquisition.

In conclusion, when the membrane is well humidified (close to the saturation value), small humidity variations do not produce any significant ohmic resistance variation, but they might produce temporary flooding (localized outside the membrane in the gas diffusion layer or catalyst layer) with consequent significant voltage drop for a very short time (typically less than $1 \mathrm{~s}$ ). This phenomenon does not represent a problem from the measurement point of view because it is easily recognizable and the measurement can be repeated after a very short time (a few seconds). On the contrary, when the membrane humidity is lower than the saturation level (a condition that is frequently adopted in practice to reduce flooding occurrence due to unexpected operating fluctuations), uncontrolled humidity variations produce a continuous ohmic resistance variation that affects impedance measurements, particularly at low frequency for the reasons explained above.

According to the equivalent circuit shown in Fig. 1, which is commonly employed to describe the linearized PEM FC behavior around an equilibrium point [1], a variation of the ohmic resistance $R_{\mathrm{ohm}}$ produces a shift of the whole impedance spectrum along the real axis, so that all the impedance measurements at different frequencies are affected by this variation, as shown in Fig. 2. Moreover, as already discussed in the previous sections, when significant variations 


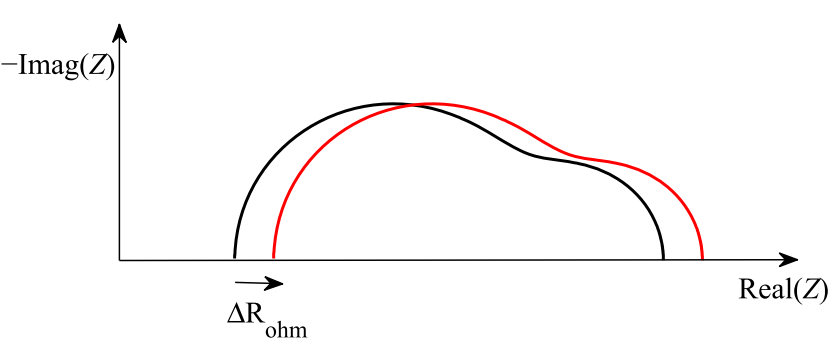

Fig. 2. Impedance spectrum shift due to an ohmic resistance variation.

occur during a single impedance measurement, the meaning of the impedance measurement itself is lost and this prevents from measuring low-frequency impedances that could provide relevant information about important processes [1], [5]. Typical values of the equivalent circuit parameters in Fig. 1 and ohmic resistance variation are reported in Section $\mathrm{V}$ for the FC employed for the experimental analysis in this paper.

\section{Proposed Method for the Compensation of OHMIC RESISTANCE VARIATIONS}

The FC ohmic resistance is generally evaluated as the highfrequency limit value of the impedance spectrum, according to the equivalent circuit of Fig. 1, or similar ones. For this reason, it can be measured via impedance measurement at a single high frequency, being the real part of the impedance at this frequency a good estimate of the ohmic resistance. This method is known as high-frequency resistance measurement [18] and it is widely used, particularly for diagnostic purposes [17], because it is simpler and extremely faster than the acquisition of the whole impedance spectrum; in addition, the perturbation signal can be generated by power converters without requiring complex and expensive instrumentation [40]. Typical employed frequencies are in the range from 1 to $10 \mathrm{kHz}$.

While such high-frequency measurement is commonly performed for diagnostic purposes, here it is proposed as a possible approach to compensate the FC instability associated with ohmic resistance variation. The high-frequency resistance measurement can be performed simultaneously with classic single-sine (or multisine) EIS analysis, by superimposing the high-frequency excitation signal to the main signal used for EIS, thus allowing us to monitor the ohmic resistance variation during low-frequency impedance measurements and eliminate the distortion that it produces on the response voltage waveform, to achieve accurate measurements also in nonstationary conditions.

It is important to note that the addition of this highfrequency component to the excitation signal can increase the overall perturbation peak-to-peak amplitude with no consequence for what concerns the linearity requirement, being the high-frequency response of the FC practically linear, as it corresponds to the ohmic resistance. The nonlinear part of the equivalent circuit (associated with activation and transport processes) acts as a short circuit in response to highfrequency current perturbations and it provides no contribution to the overall voltage waveform. Therefore, the high-frequency resistance measurement performed simultaneously with EIS does not require to worsen the signal-to-noise ratio of the signals used for impedance spectroscopy, thus it does not increase the measured impedance uncertainty. Moreover, for the same reason of linear behavior, the signal-to-noise ratio of the high-frequency signal can be even higher than the low-frequency one, thus allowing us to obtain quite accurate resistance measurements.

Another important advantage of high-frequency resistance measurement is the very small time resolution, arising from the small period of the high-frequency excitation signal, which allows a very good monitoring of ohmic resistance variations, typically occurring on time scales of several seconds.

The only requirement for accurate measurements is that the maximum frequency used for EIS is much lower (let us say, two decades below) than the high frequency used for ohmic resistance measurement, so that no significant leakage errors arise when applying the Fourier transform to a single period (or a few periods) of the high-frequency signal component. However, this requirement does not represent a limitation for the proposed method, because the ohmic resistance compensation is only necessary for low-frequency impedance measurements, whose acquisition time is long enough to allow the ohmic resistance to change. On the contrary, impedances at higher frequencies can be accurately measured without the proposed compensation technique, since the system can be considered in quasi-stationary conditions on these short time scales.

For the experimental analysis reported in this paper, the proposed method was applied for single-sine impedance measurements at 10 and $100 \mathrm{mHz}$, using a $1-\mathrm{kHz}$ sine wave for ohmic resistance measurements. Another harmonic component at $40 \mathrm{~Hz}$ was added to the excitation signal to experimentally verify that the instabilities appearing in the $\mathrm{FC}$ voltage are only due to ohmic resistance variations and that they only produce a shift of the whole impedance spectrum along the real axis, in agreement with the approximation of the equivalent circuit in Fig. 1 (or any other circuit in which $R_{\text {ohm }}$ is a series resistance). If this is true, no significant variation is expected in the imaginary part of lower frequency impedances. The value of $40 \mathrm{~Hz}$ was chosen because it is close to the expected frequency corresponding to the maximum of the impedance imaginary part, for the FC under test.

Because of the $40-\mathrm{Hz}$ component in the excitation signal, the chosen time window for the $1-\mathrm{kHz}$ and $40-\mathrm{Hz}$ impedance calculation is $100 \mathrm{~ms}$, containing 100 and 4 periods of the $1-\mathrm{kHz}$ and $40-\mathrm{Hz}$ harmonics, respectively. This choice allows to use a fourth-order window to reduce leakage errors in the Fourier transform, and it guarantees a proper time resolution (100 ms) for the ohmic resistance measurement. Such ohmic resistance measurement together with the FC current measurement allows, then, to calculate the ohmic voltage drop $R_{\mathrm{ohm}} i$, which can be removed from the measured voltage waveform; so, it is possible to calculate the impedance of the low-frequency part of the equivalent circuit without the ohmic resistance contribution that has been independently measured. In symbols

$$
Z_{\text {If }}(j \omega)=-\frac{V^{\prime}(j \omega)}{I_{\text {cell }}(j \omega)}
$$




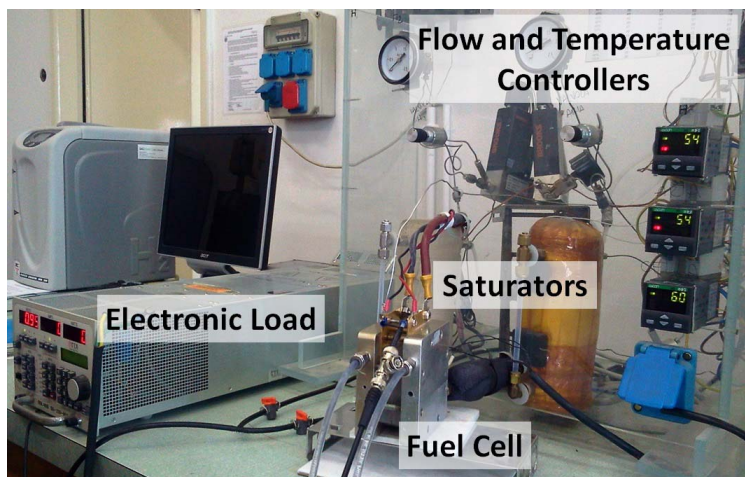

Fig. 3. Photograph of the experimental setup.

TABLE I

FC EQUivalent Circuit PARAMETERS

\begin{tabular}{|c|c|c|c|c|}
\hline$R_{\mathrm{ohm}}$ & $R_{\text {act }}$ & $C_{\text {act }}$ & $R_{\text {trans }}$ & $C_{\text {trans }}$ \\
\hline $4.7 \mathrm{~m} \Omega$ & $9.6 \mathrm{~m} \Omega$ & $6.9 \mathrm{~F}$ & $4.2 \mathrm{~m} \Omega$ & $0.24 \mathrm{~F}$ \\
\hline
\end{tabular}

where

$$
v^{\prime}(t)=v_{\text {cell }}(t)+R_{\text {ohm }}(t) i_{\text {cell }}(t) .
$$

The signs in (1) and (2) are in agreement with the voltage and current sign conventions shown in Fig. 1.

\section{EXPERIMENTAL RESULTS}

The experimental validation of the proposed measurement method was carried out on a single PEM FC (FC Technologies) composed of commercial materials. In more details, a commercial Nafion 212 membrane with $23-\mathrm{cm}^{2}$ area and $50-\mu \mathrm{m}$ thickness was employed as electrolyte, together with a commercial gas diffusion electrode reference sample (E-TEK LT140). A photograph of the experimental setup is shown in Fig. 3.

The cell was fed with pure hydrogen and air at the anode and cathode, respectively, with constant flow rates of $0.2 \mathrm{Nl} / \mathrm{min}$ for hydrogen and $1.0 \mathrm{Nl} / \mathrm{min}$ for air (corresponding to a stoichiometric ratio of 1.2/2.0 A/C @ $1 \mathrm{~A} / \mathrm{cm}^{2}$ ), controlled by calibrated flow meters. The input gas humidity was controlled by saturators: the gas was fully humidified at a controllable temperature, so that the desired relative humidity was obtained when the gas entered the cell, whose temperature was kept constant at $60{ }^{\circ} \mathrm{C}$. Equal relative humidities were set for hydrogen and air, in particular $60 \%$ or $100 \%$ for the experimental analysis reported here. Typical FC parameters in the operating conditions chosen for the tests are summarized in Table I, with reference to the equivalent circuit of Fig. 1.

The FC output current was imposed by an electronic load (TDI RBL488-50-150-800) according to a reference signal generated by a 16-bit multifunction data acquisition (DAQ) system (NI 6251), containing both dc and ac components. The same DAQ system was also employed to acquire the FC voltage and the current measurement provided by the electronic load as a voltage signal proportional to the FC current. Both signals were acquired with $5 \cdot 10^{5}$ samples/s sampling frequency. The FC impedance was then calculated as

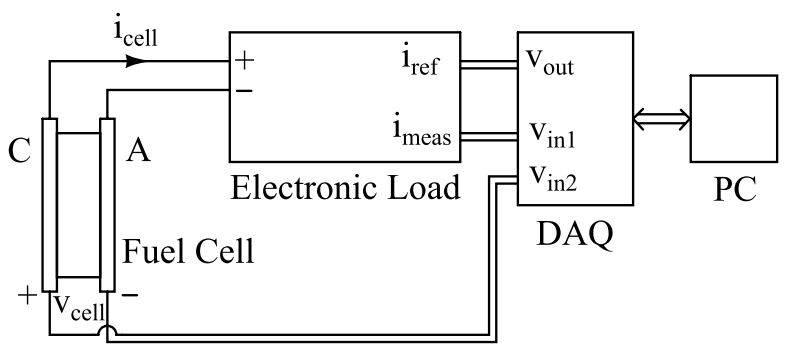

Fig. 4. Diagram of the measurement circuit.
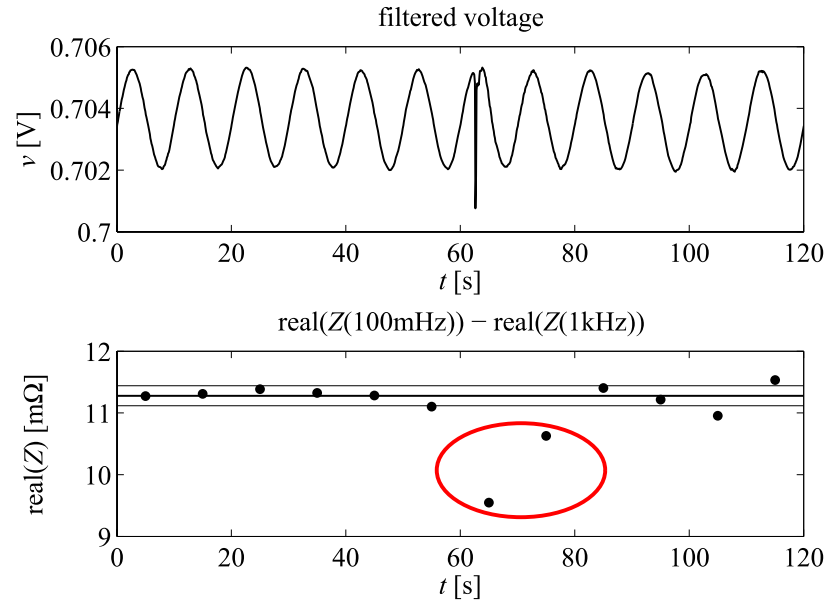

Fig. 5. Top: measured voltage response to a $100-\mathrm{mHz}$ current perturbation at $100 \%$ relative humidity. Bottom: low-frequency equivalent resistance. Horizontal lines: the mean value and the standard deviation band calculated excluding the outliers due to the voltage spike (circled points).

complex ratio of the voltage and current spectra, which in turn were obtained by applying the discrete Fourier transform on these sampled signals. A diagram of the measurement circuit is shown in Fig. 4.

The voltage and current uncertainties can be estimated from the DAQ and electronic load specifications, which provide accuracy and resolution. In the measurement ranges employed for the tests reported here, the main voltage uncertainty contribution arises from the DAQ accuracy $(1.8 \mathrm{mV})$, providing a $1.1-\mathrm{mV}$ uncertainty, while the main contribution for current is given by the load accuracy $(100 \mathrm{~mA})$, providing a $58-\mathrm{mA}$ uncertainty.

All tests were performed at a medium dc $(8.8 \mathrm{~A}$, corresponding to $0.38 \mathrm{~A} / \mathrm{cm}^{2}$ ), to avoid high water production from the chemical reaction, so that the membrane humidity could be better controlled by the input gas humidity. As anticipated, two humidity levels were chosen, $60 \%$ and $100 \%$. The ac perturbation contained a low-frequency component $(10$ or $100 \mathrm{mHz}$ ) with 200-mA peak-to-peak amplitude and two high-frequency components $(40 \mathrm{~Hz}$ and $1 \mathrm{kHz})$, each of them with $500-\mathrm{mA}$ peak-to-peak amplitude.

The first test was performed at $100 \%$ relative humidity. The FC voltage response to a $100-\mathrm{mHz}$ current perturbation is shown in Fig. 5, together with the impedance values of the low-frequency circuit for each period (corresponding to the sum of transport and activation resistances), while the 


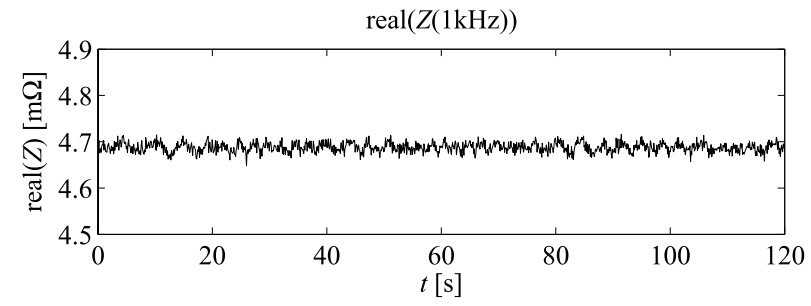

$\operatorname{imag}(Z(40 \mathrm{~Hz}))$

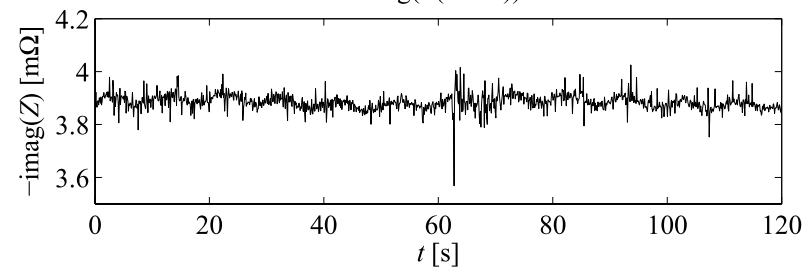

Fig. 6. Impedance real and imaginary parts at $1 \mathrm{kHz}$ and $40 \mathrm{~Hz}$, respectively, when a $100-\mathrm{mHz}$ current perturbation is applied at $100 \%$ relative humidity.

ohmic resistance and the imaginary part of the impedance at $40 \mathrm{~Hz}$ are shown in Fig. 6. The impedance of the lowfrequency circuit is calculated as the difference between the low-frequency impedance and a constant ohmic resistance (the mean value of the measurements in Fig. 6). It can be observed that no significant distortions appear in the voltage waveform, in agreement with the quite stable ohmic resistance, except for a significant spike. As discussed in Section III, this spike is probably due to a temporary flooding of the membrane. It obviously affects the impedance measurement in the period containing the spike and in the following one, but this does not represent a problem from the measurement point of view because such measurements can be easily recognized and discarded. The standard deviation of the other 10 measurements shown in Fig. 5 is $0.16 \mathrm{~m} \Omega$, meaning that the type-A uncertainty is reasonably low in this case.

On the contrary, a very different scenario appears at $60 \%$ relative humidity. The results obtained with a $100-\mathrm{mHz}$ current perturbation are shown in Figs. 7 and 8, with the same meaning of Figs. 5 and 6 . In this case, significant ohmic resistance variations (more than $0.2-\mathrm{m} \Omega$ peak-to-peak) occur continuously, while the impedance imaginary part at $40 \mathrm{~Hz}$ is approximately constant, and consequently significant distortions are visible in the voltage waveform. If the impedance of the low-frequency circuit is calculated from the measured voltage considering a constant ohmic resistance as done above, the standard deviation of the experimental data is quite high $(1.15 \mathrm{~m} \Omega)$, while it decreases to a lower value $(0.59 \mathrm{~m} \Omega)$ if the ohmic resistance variations are compensated in the time domain according to the proposed method. This means that the type-A uncertainty evaluated in the first case would be overestimated.

It should be noted that, in this case, the voltage waveform distortion appears at frequencies smaller than the excitation frequency; therefore, an improvement of measurement results could be obtained also using proper windows to reduce leakage errors, although this technique would significantly increase the measurement time.

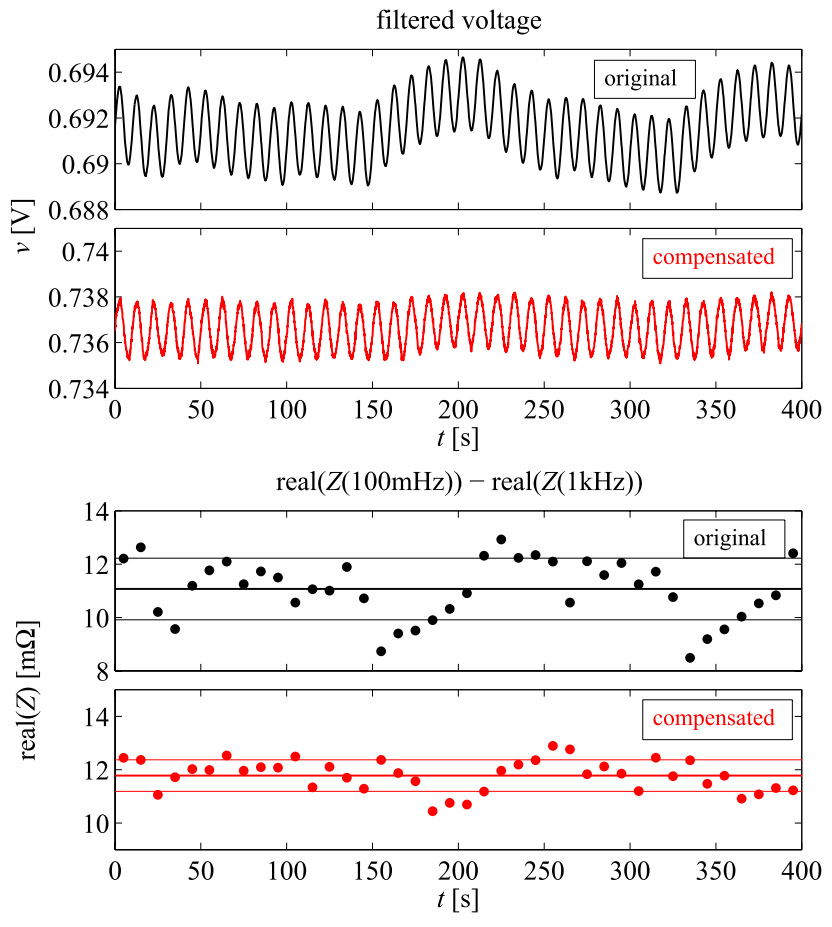

Fig. 7. Top: measured (black) voltage response to a $100-\mathrm{mHz}$ current perturbation at $60 \%$ relative humidity and compensated (red) waveform without ohmic voltage drop. Bottom: low-frequency equivalent resistance calculated from the original (black) and compensated (red) data. Horizontal lines: the mean value and the standard deviation band.

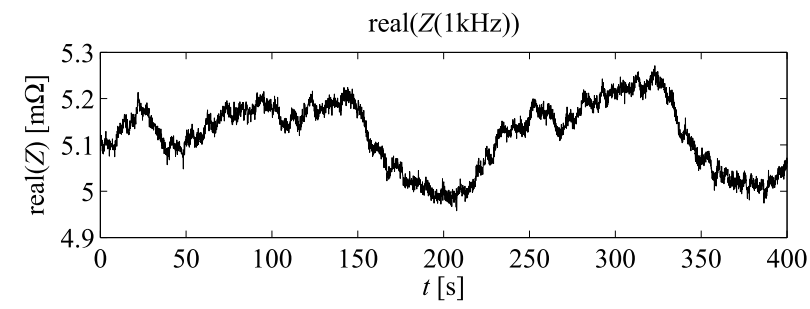

$\operatorname{imag}(Z(40 \mathrm{~Hz}))$

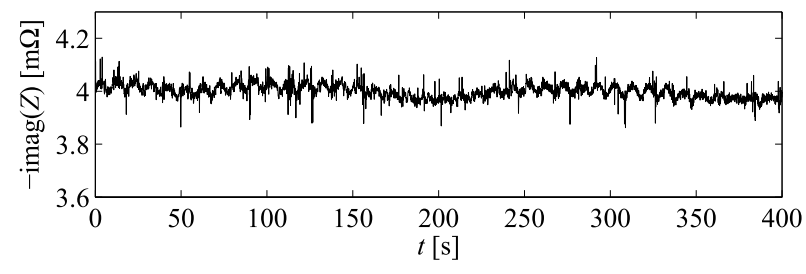

Fig. 8. Impedance real and imaginary parts at $1 \mathrm{kHz}$ and $40 \mathrm{~Hz}$, respectively, when a $100-\mathrm{mHz}$ current perturbation is applied at $60 \%$ relative humidity.

On the contrary, when a $10-\mathrm{mHz}$ current perturbation is considered (Figs. 9 and 10), the ohmic resistance variations occur on a time scale comparable with the period of the excitation signal, meaning that there is no more frequency separation between the applied perturbation and the ohmic resistance variation. In this case, completely meaningless results appear from the calculation of the impedance of the low-frequency circuit from the measured waveform, and neither the use of windows would improve the results. However, the proposed compensation technique provides a stable voltage waveform and almost constant impedance measurements. The standard 

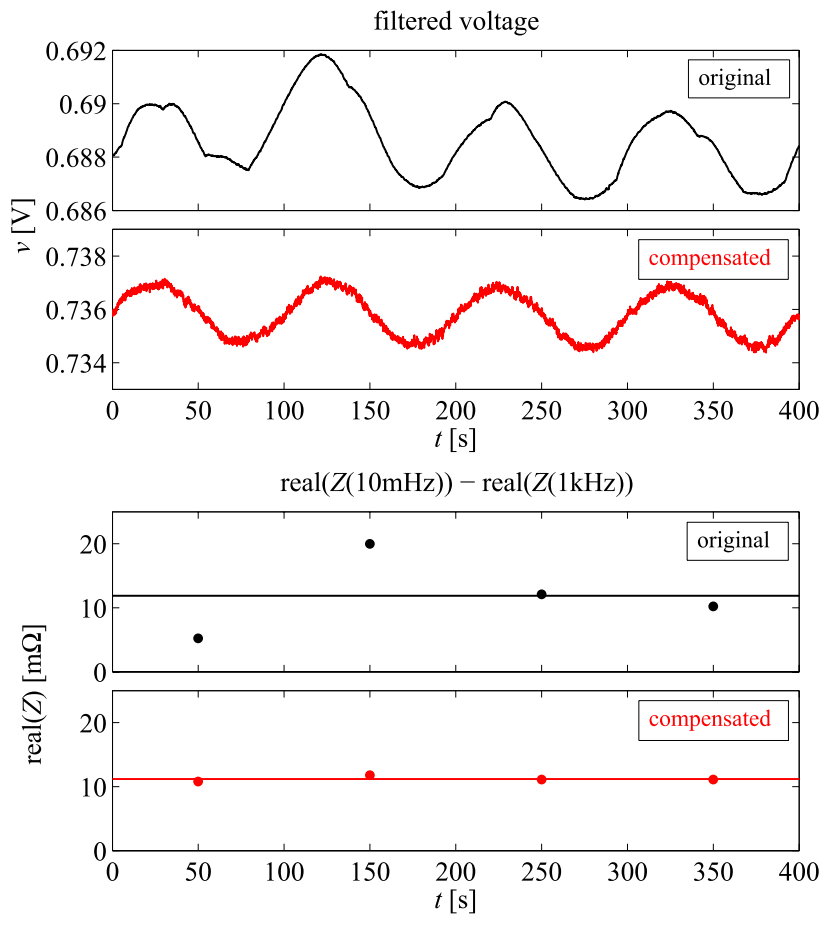

Fig. 9. Top: measured (black) voltage response to a $10-\mathrm{mHz}$ current perturbation at $60 \%$ relative humidity and compensated (red) waveform without ohmic voltage drop. Bottom: low-frequency equivalent resistance calculated from the original (black) and compensated (red) data. Horizontal line: the mean value.
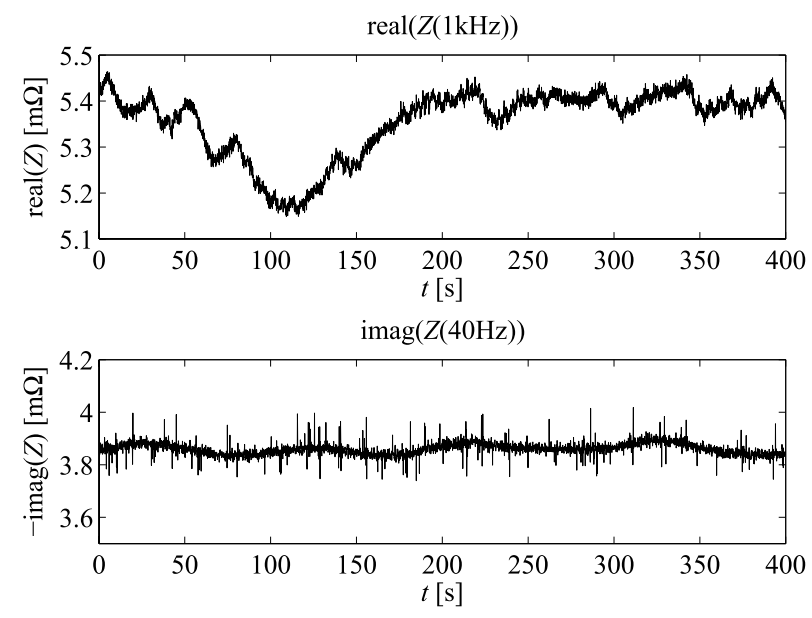

Fig. 10. Impedance real and imaginary parts at $1 \mathrm{kHz}$ and $40 \mathrm{~Hz}$, respectively, when a $10-\mathrm{mHz}$ current perturbation is applied at $60 \%$ relative humidity.

deviation has poor meaning in this case because only four values are considered, but significant information can be retrieved from the maximum difference between two impedance measurements: 14.76 and $0.97 \mathrm{~m} \Omega$ before and after compensation, respectively. Again, the type-A uncertainty calculated in the first case would be greatly overestimated.

\section{CONCLUSION}

The problem of EIS measurements on PEM FCs in nonstationary conditions was reapproached, focusing on humidityrelated instabilities that cause significant variations of the FC equivalent ohmic resistance during low-frequency impedance measurements.

To correctly interpret impedance measurements affected by such instabilities, a new method was proposed and validated on a single PEM FC. The method is based on the continuous measurement of the ohmic resistance by means of a highfrequency sinusoidal perturbation superimposed to the excitation signal, so that the time-varying ohmic voltage drop can be calculated and removed from the output voltage waveform in the time domain. As a result, the waveform distortion due to the ohmic resistance variation is eliminated and the frequency response of the time-invariant part of the FC equivalent circuit can be then accurately evaluated with classic Fourier transform analysis.

Furthermore, this method allows to evaluate the correlation between the ohmic resistance and the low-frequency impedance, important to correctly estimate the type-A uncertainty of the low-frequency parameters identified from the impedance spectrum. Such evaluation allowed to extend the useful frequency range for EIS down to $10 \mathrm{mHz}$, with an estimated uncertainty of the low-frequency parameters, which was about one order of magnitude lower than the estimate obtained with the classic approach.

\section{REFERENCES}

[1] X. Z. Yuan, C. Song, H. Wang, and J. Zhang, Electrochemical Impedance Spectroscopy in PEM Fuel Cells. London, U.K.: Springer-Verlag, 2010.

[2] E. Barsoukov and J. R. Macdonald, Impedance Spectroscopy, 2nd ed. Hoboken, NJ, USA: Wiley, 2005.

[3] B. Y. Chang and S. M. Park, "Electrochemical impedance spectroscopy," Annu. Rev. Anal. Chem., vol. 3, no. 1, pp. 207-229, 2010.

[4] P. M. Gomadam and J. W. Weidner, "Analysis of electrochemical impedance spectroscopy in proton exchange membrane fuel cells," Int. J. Energy Res., vol. 29, no. 12, pp. 1133-1151, 2005.

[5] A. M. Dhirde, N. V. Dale, H. Salehfar, M. D. Mann, and T. H. Han, "Equivalent electric circuit modeling and performance analysis of a PEM fuel cell stack using impedance spectroscopy," IEEE Trans. Energy Convers., vol. 25, no. 3, pp. 778-786, Sep. 2010.

[6] N. Wagner, "Characterization of membrane electrode assemblies in polymer electrolyte fuel cells using AC impedance spectroscopy," J. Appl. Electrochem., vol. 32, no. 8, pp. 859-863, 2002.

[7] M. A. Danzer and E. P. Hofer, "Analysis of the electrochemical behaviour of polymer electrolyte fuel cells using simple impedance models," J. Power Sour, vol. 190, no. 1, pp. 25-33, 2009.

[8] J. M. Le Canut, R. Latham, W. Mérida, and D. A. Harrington, "Impedance study of membrane dehydration and compression in proton exchange membrane fuel cells," J. Power Sour., vol. 192, no. 2, pp. 457-466, 2009.

[9] G. Dotelli, L. Omati, P. Gallo Stampino, P. Grassini, and D. Brivio, "Investigation of gas diffusion layer compression by electrochemical impedance spectroscopy on running polymer electrolyte membrane fuel cells," J. Power Sour., vol. 196, pp. 8955-8966, Nov. 2011.

[10] L. Omati, P. Gallo Stampino, G. Dotelli, D. Brivio, and P. Grassini, "Operative conditions effect on PEM-FC performance by in-situ and ex-situ analysis of gas diffusion media with different bulk textile structure," Int. J. Hydrogen Energy, vol. 36, no. 13, pp. 8053-8062, 2011.

[11] P. Gallo Stampino, S. Latorrata, D. Molina, S. Turri, M. Levi, and G. Dotelli, "Investigation of hydrophobic treatments with perfluoropolyether derivatives of gas diffusion layers by electrochemical impedance spectroscopy in PEM-FC," Solid State Ion., vol. 216, pp. 100-104, May 2012.

[12] R. Ferrero, M. Marracci, M. Prioli, and B. Tellini, "Simplified model for evaluating ripple effects on commercial PEM fuel cell," Int. J. Hydrogen Energy, vol. 37, no. 18, pp. 13462-13469, 2012.

[13] R. Ferrero, M. Marracci, and B. Tellini, "Single PEM fuel cell analysis for the evaluation of current ripple effects," IEEE Trans. Instrum. Meas., vol. 62, no. 5, pp. 1058-1064, May 2013. 
[14] X. Yuan, H. Wang, J. C. Sun, and J. Zhang, "AC impedance technique in PEM fuel cell diagnosis-A review," Int. J. Hydrogen Energy, vol. 32, pp. 4365-4380, Dec. 2007.

[15] N. Fouquet, C. Doulet, C. Nouillant, G. Dauphin-Tanguy, and B. OuldBouamama, "Model based PEM fuel cell state-of-health monitoring via AC impedance measurements," J. Power Sour., vol. 159, no. 2, pp. 905-913, 2006.

[16] W. Mérida, D. A. Harrington, J. M. Le Canut, and G. McLean, "Characterisation of proton exchange membrane fuel cell (PEMFC) failures via electrochemical impedance spectroscopy," J. Power Sour. vol. 161, no. 1, pp. 264-274, 2006.

[17] T. Kurz, A. Hakenjos, J. Krämer, M. Zedda, and C. Agert, "An impedance-based predictive control strategy for the state-of-health of PEM fuel cell stacks," J. Power Sour., vol. 180, no. 2, pp. 742-747, 2008.

[18] K. R. Cooper and M. Smith, "Electrical test methods for on-line fuel cell ohmic resistance measurement," J. Power Sour., vol. 160, no. 2, pp. 1088-1095, 2006.

[19] R. Ferrero, G. Dotelli, P. Gallo Stampino, and S. Latorrata, "Discussion of critical measurement issues of impedance spectroscopy on PEM fuel cells," in Proc. IEEE AMPS, Aachen, Germany, Sep. 2012, pp. 97-102.

[20] G. S. Popkirov, "Fast time-resolved electrochemical impedance spectroscopy for investigations under nonstationary conditions," Electrochim. Acta, vol. 41, nos. 7-8, pp. 1023-1027, 1996.

[21] T. Breugelmans, J. Lataire, T. Muselle, E. Tourwé, R. Pintelon, and A. Hubin, "Odd random phase multisine electrochemical impedance spectroscopy to quantify a non-stationary behaviour: Theory and validation by calculating an instantaneous impedance value," Electrochim. Acta, vol. 76, pp. 375-382, Aug. 2012.

[22] M. Ordonez, M. O. Sonnaillon, J. E. Quaicoe, and M. T. Iqbal, "An embedded frequency response analyzer for fuel cell monitoring and characterization," IEEE Trans. Ind. Electron., vol. 57, no. 6, pp. 1925-1934, Jun. 2010.

[23] A. E. Novik, M. N. Surdu, and L. P. Sheremet, USSR Patent 637679 , Dec. 15, 1978.

[24] G. S. Popkirov and R. N. Schindler, "A new impedance spectrometer for the investigation of electrochemical systems," Rev. Sci. Instrum., vol. 63, no. 11 , pp. 5366-5372, 1992.

[25] K. Darowicki, J. Orlikowski, and G. Lentka, "Instantaneous impedance spectra of a non-stationary model electrical system," J. Electroanal. Chem., vol. 486, no. 2, pp. 106-110, 2000.

[26] E. Van Gheem, R. Pintelon, J. Vereecken, J. Schoukens, A. Hubin, P. Verboven, et al., "Electrochemical impedance spectroscopy in the presence of non-linear distortions and non-stationary behaviour: Part I: Theory and validation," Electrochim. Acta, vol. 49 , pp. 4753-4762, Oct. 2004.

[27] Y. Van Ingelgem, E. Tourwé, O. Blajiev, R. Pintelon, and A. Hubin, "Advantages of odd random phase multisine electrochemical impedance measurements," Electroanalysis, vol. 21, no. 6, pp. 730-739, 2009.

[28] B. Sanchez, G. Vandersteen, R. Bragos, and J. Schoukens, "Basics of broadband impedance spectroscopy measurements using periodic excitations," Meas. Sci. Technol., vol. 23, no. 10, pp. 105501-1-105501-14, 2012.

[29] C. Gabrielli, F. Huet, and M. Keddam, "Comparison of sine wave and white noise analysis for electrochemical impedance measurements," J. Electroanal. Chem., vol. 335, nos. 1-2, pp. 33-53, 1992.

[30] P. A. Lindahl, M. A. Cornachione, and S. R. Shaw, "A time-domain least squares approach to electrochemical impedance spectroscopy," IEEE Trans. Instrum. Meas., vol. 61, no. 12, pp. 3303-3311, Dec. 2012.

[31] M. Urquidi-Macdonald, S. Real, and D. D. Macdonald, "Applications of Kramers-Kronig transforms in the analysis of electrochemical impedance data-III. Stability and linearity," Electrochim. Acta, vol. 35 no. 10 , pp. $1559-1566,1990$.

[32] P. Agarwal, M. E. Orazem, and L. H. Garcia-Rubio, "Measurement models for electrochemical impedance spectroscopy I. Demonstration of applicability," J. Electrochem. Soc., vol. 139, no. 7, pp. 1917-1927, 1992.

[33] G. S. Popkirov and R. N. Schindler, "Validation of experimental data in electrochemical impedance spectroscopy," Electrochim. Acta, vol. 38, no. 7, pp. 861-867, 1993.

[34] Z. Stoynov, "Nonstationary impedance spectroscopy," Electrochim. Acta, vol. 38, no. 14, pp. 1919-1922, 1993.
[35] C. A. Schiller, F. Richter, E. Gülzow, and N. Wagner, "Validation and evaluation of electrochemical impedance spectra of systems with states that change with time," Phys. Chem. Chem. Phys., vol. 3, no. 3, pp. 374-378, 2001.

[36] A. Dupuis, "Proton exchange membranes for fuel cells operated at medium temperatures: Materials and experimental techniques," Progr. Mater. Sci., vol. 56, no. 3, pp. 289-327, 2011.

[37] W. Dai, H. Wang, X. Yuan, J. J. Martin, D. Yang, J. Qiao, et al. "A review on water balance in the membrane electrode assembly of proton exchange membrane fuel cells," Int. J. Hydrogen Energy, vol. 34, no. 23, pp. 9461-9478, 2009.

[38] C. H. Park, C. H. Lee, M. D. Guiver, and Y. M. Lee, "Sulfonated hydrocarbon membranes for medium-temperature and low-humidity proton exchange membrane fuel cells (PEMFCs)," Progr. Polymer Sci., vol. 36, no. 11, pp. 1443-1498, 2011.

[39] K. Wiezell, P. Gode, and G. Lindbergh, "Steady-state and EIS investigations of hydrogen electrodes and membranes in polymer electrolyte fuel cells," J. Electrochem. Soc., vol. 153, no. 4, pp. A749-A758, 2006.

[40] G. Dotelli, R. Ferrero, P. Gallo Stampino, and S. Latorrata, "Inverter ripple as a diagnostic tool for ohmic resistance measurements on PEM fuel cells," in Proc. IEEE AMPS, Aachen, Germany, Sep. 2013, pp. $156-161$.

Giovanni Dotelli was born in Piacenza, Italy, in 1964. He received the M.Sc degree in chemical engineering and the Ph.D. degree in materials engineering from Politecnico di Milano, Milan, Italy, in 1989 and 1993, respectively.

$\mathrm{He}$ has been an Associate Professor of materials science with the Department of Chemistry, Materials, and Chemical Engineering "G.Natta," Politecnico di Milano, since 2001. He is currently with the Laboratory of Materials for Energy and Environment. His current research interests include the materials for energy- and environmental-related sustainable technologies.

Roberto Ferrero (S'10-M'14) was born in Milan, Italy, in 1986. He received the M.Sc. and Ph.D. degrees in electrical engineering from Politecnico di Milano, Milan, in 2009 and 2013, respectively.

He has been a Temporary Research Fellow with the Department of Energy, Systems, Land and Constructions, University of Pisa, Pisa, Italy, since 2013. His current research interests include electrical measurements particularly applied to electromagnetic launchers and fuel cells.

Paola Gallo Stampino was born in Busto Arsizio, Italy, in 1974. She received the M.Sc. degree in material science from the University of Milano-Bicocca, Milan, Italy, and the Ph.D. degree in materials engineering from Politecnico di Milano, Milan, in 2002 and 2007, respectively.

She has been an Assistant Professor with the Department of Chemistry, Materials, and Chemical Engineering "G.Natta," Politecnico di Milano, since 2008. Her current research interests include the synthesis and characterization of materials for energy and environment.

Saverio Latorrata was born in Taranto, Italy, in 1984. He received the M.Sc. degree in chemical engineering from Politecnico di Milano, Milan, Italy, in 2008, where he has been pursuing the Ph.D. degree in materials engineering with the Department of Chemistry, Materials and Chemical Engineering, since 2011.

He was a Research Fellow with the Department of Chemistry, Materials and Chemical Engineering "G.Natta," Politecnico di Milano, in 2010. His current research interests include the development and characterization of innovative materials for polymer electrolyte membrane fuel cells. 